\title{
High-Gain and Low-RCS Linear Polarization F-P Resonant Cavity Antenna Based on Metasurface
}

\author{
Jia LU, Xiangyu CAO, Jun GAO, HuanHuan YANG, Liaori JIDI, Kun GAO \\ Information and Navigation College of Air Force Engineering University, Xi'an, Shaanxi, 710077, China \\ lujia4251@foxmail.com, xiangyucaokdy@163.com \\ Submitted June 17, 2021 / Accepted September 25, 2021
}

\begin{abstract}
Fabry-Perot (F-P) resonant cavity antenna is a high-gain antenna, which can increase the gain of microstrip antenna significantly, without a complicated feed network. It has a simple structure and it is easy to process. In this paper, the polarization grid structure and the zigzag structure are combined to design a linear-circular polarization conversion metasurface unit. This unit can convert the linearly polarized incident wave that is perpendicular to the polarization grid into circularly polarized transmitted wave in the range of $9.26 \sim 10.84 \mathrm{GHz}$. The unit and its mirror image unit are arranged in a checkerboard shape as a metasurface and placed above the microstrip antenna. Thus a linear polarized $F-P$ resonant cavity antenna with high gain and low radar cross section (RCS) is proposed. In order to verify the performance of the antenna, the F-P resonant cavity antenna was processed and measured. The measured results and simulated results have shown good consistency. Compared with the original microstrip antenna, the polarization purity of the F-P resonator cavity antenna is enhanced in the range of 9.55 9.85 GHz, and the gain is improved in the working frequency range of 9.50 10.04 GHz with a maximum increase of $5.55 \mathrm{~dB}$, and the RCS reduction was achieved in the range of 9.47 11.97 GHz with a maximum reduction of $17.47 \mathrm{~dB}$.
\end{abstract}

\section{Keywords}

F-P resonant cavity antenna, metasurface, high gain, low RCS

\section{Introduction}

Nowadays, with the continuous development of radar, beyond-visual-range warfare has gradually become one of the main modes of modern warfare. Therefore, all the countries in the world attach great importance to the research and application of radar stealth technology. RCS is an important indicator that reflects the performance of radar stealth. With the application of new stealth technologies such as frequency selective surface (FSS) and waveabsorbing coatings, the RCS of aircrafts and ships and other weapon platforms has become very low. However, as the electromagnetic open window on the stealth platform, a large number of antennas become the main contribution of its scattering. So it is very necessary to reduce the RCS of the antenna.

Metasurface is a two-dimensional metamaterial structure that is arranged by sub-wavelength units periodically or quasi-periodically. By designing the unit structure and its arrangement, the electromagnetic (EM) wave characteristics can be modulated [1]. It has significant advantages in polarization control [4], [5]. In recent years, design of antenna based on metasurfaces has become a very important direction in antenna research to improve performance of antenna, such as increasing gain and reducing RCS [6-10]. In [11] and [12], the RCS reduction of the antenna is successfully achieved by phase cancellation between different antenna elements. It is a method to improve the performance of antenna by using metasurface coating on top of antenna [13], [14]. In [15] and [16], F-P resonant cavity antennas are designed to improve gain and reduce RCS.

In this paper, a transmissive linear-circular polarization conversion metasurface unit is designed. The unit and its mirror image unit are arranged in a checkerboard shape as a metasurface and placed above the microstrip antenna. The metasurface and the antenna ground plate form an F-P resonant cavity, so a linear polarized F-P resonant cavity antenna with high gain and low RCS is designed. Compared with the original antenna, the designed F-P resonator cavity antenna has a higher gain, higher polarization purity and lower RCS. Finally, the antenna was processed and has been measured in a microwave anechoic chamber. The simulation results are in good agreement with the measurement results.

\section{Theoretical Analysis and Unit Design}

\subsection{The Principle of the F-P Resonant Cavity Antenna}

The F-P resonator cavity antenna is a high-gain antenna. It has a cladding structure with partial reflection characteristics which is placed on the top of the antenna, as shown in Fig. 1. The EM wave radiated by the feed is incident on the partially reflective surface (PRS) at $\theta$, part of 


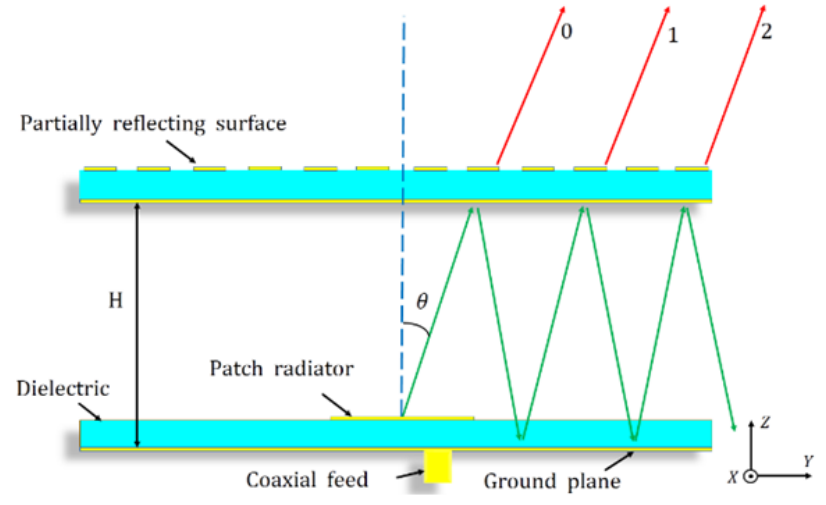

Fig. 1. Schematic of the F-P resonator cavity antenna.

wave is transmitted through the PRS, and the other part is reflected back into the cavity by the metasurface. Then the reflected EM wave will be totally reflected by the ground plane of antenna at $\theta$. The EM wave will be reflected and transmitted several times in the resonator cavity. When the resonant condition is satisfied, the transmitted EM wave will be stack in same phase on the outside of the PRS, thus significantly improving the gain of the antenna [15-17].

Assuming that the transmitting coefficient and reflection coefficient of the metasurface can be expressed as

$$
t=T \mathrm{e}^{\mathrm{j} \theta_{1}}, r=R \mathrm{e}^{\mathrm{j} \varphi_{\text {PRS }}} .
$$

Then the transmission coefficient of the EM wave that directly penetrates the metasurface by the feed radiation can be expressed as

$$
t_{0}=t
$$

When the EM wave is reflected by the metasurface once, the transmission coefficient can be calculated as

$$
\begin{aligned}
t_{1} & =\operatorname{tr} \mathrm{e}^{-2 \mathrm{j} k h / \cos \theta+2 \mathrm{j} k h \tan \theta \sin \theta+\mathrm{j} \varphi_{\mathrm{GND}}} \\
& =\operatorname{tr} \mathrm{e}^{-2 \mathrm{j} k h \cos \theta+\mathrm{j} \varphi_{\mathrm{GND}}} .
\end{aligned}
$$

In the above equation, $\varphi_{\mathrm{GND}}$ is the reflection phase of the antenna bottom metal plate, $k=2 \pi / \lambda$ is the propagation constant, and $\lambda$ is the wavelength. It can be deduced that the transmission coefficient of the resonator cavity after $n$ times reflection can be expressed as (4). Therefore, the sum of all transmission coefficients along the direction of $\theta$ can be calculated as (5):

$$
\begin{gathered}
t_{n}=t r^{n} \mathrm{e}^{-2 \mathrm{j} n k h \cos \theta+\mathrm{j} n \varphi_{\mathrm{GND}}}, \\
t_{\text {total }}=\sum_{n=0}^{\infty} t_{n}=\frac{t}{1-r \mathrm{e}^{-2 \mathrm{j} k h \cos \theta+\mathrm{j} n \varphi_{\mathrm{GND}}} .}
\end{gathered}
$$

According to the law of conservation of energy, the total transmission coefficient of power is given by (6):

$$
\begin{gathered}
D=t_{\text {total }} t_{\text {total }}^{*}=\frac{1-R^{2}}{1+R^{2}-2 R \cos \Phi}, \\
\Phi=\frac{4 \pi}{\lambda} h \cos \theta-\varphi_{\mathrm{PRS}}-\varphi_{\mathrm{GND}} .
\end{gathered}
$$

In (7), $\varphi_{\mathrm{PRS}}$ is the reflection phase of the PRS, and $\varphi_{\mathrm{GND}}$ is the reflection phase of the bottom metal plate. When $\cos \Phi=1$, the transmission coefficient of power can get the maximum value which can be calculated as

$$
D_{\max }=\frac{1-R^{2}}{1+R^{2}-2 R}=\frac{1+R}{1-R} .
$$

It can be seen that $D_{\max }$ is positive correlation with the reflection amplitude of the PRS. So the larger amplitude it has the higher antenna gain it achieves. Considering that the maximum radiation direction of the antenna is $\theta=0$, the antenna resonance condition can be expressed as (9). When $\varphi_{\mathrm{GND}}=\pi$, equation (9) can be expressed as (10)

$$
\begin{gathered}
H=\frac{\lambda}{4 \pi}\left(\varphi_{\mathrm{PRS}}+\varphi_{\mathrm{GND}}\right)+\frac{\lambda}{2} N, N=0,1,2, \ldots \\
H=\frac{\lambda}{4 \pi}\left(\varphi_{\mathrm{PRS}}+\pi\right)+\frac{\lambda}{2} N, N=0,1,2, \ldots
\end{gathered}
$$

\subsection{Design of Metasurface Unit}

Circularly polarized waves can be generated using metasurface [18]. In [19], the metal gap structure was used to generate circularly polarized waves. Cutting away corners is also a common method to realize circular polarization of microstrip antenna. According to this theory, this paper adopts the structure that one corner of the square metal patch is cut away and the opposite corner is cut into zigzag to realize the conversion from linear polarization to circular polarization, as shown in Fig. 2(a). In order to explain the mechanism of generating circular polarization, an equivalent circuit is used for analysis. The part of the red dashed frame in Fig. 2(b) is regarded as a unit, and the transmitted wave can be decomposed into two orthogonal components. If the corner of the unit is not cut away, as shown in Fig. 2(c), the two orthogonal components will produce the same impedance, and the impedance can be expressed as

$$
Z=2 R+2 \mathrm{j} \omega L+\frac{1}{\mathrm{j} \omega C}=R^{\prime}+\mathrm{j} X^{\prime} .
$$

In the above equation, $R$ and $L$ respectively represent the resistance and inductance of each unit, $C$ represents the capacitance generated by the gap between adjacent and opposite units. When the unit is truncated, the structure is no longer symmetrical, $\mathbf{E}_{1}$ and $\mathbf{E}_{2}$ will produce different impedance:

$$
\begin{aligned}
& Z_{1}=R_{1}^{\prime}+\mathrm{j} X_{1}^{\prime}, \\
& Z_{2}=R_{2}^{\prime}+\mathrm{j} X_{2}^{\prime} .
\end{aligned}
$$

Changing the size of the corner can produce different impedance, when $\left|Z_{1}\right|=\left|Z_{2}\right|$ and $\angle Z_{1}-\angle Z_{2}= \pm \pi / 2$, there will be $\left|E_{1}\right|=\left|E_{2}\right|$ and $\angle E_{1}-\angle E_{2}= \pm \pi / 2$, and the conversion from linear polarization to circular polarization will be achieved. 


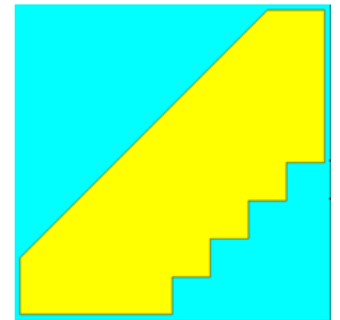

(a)

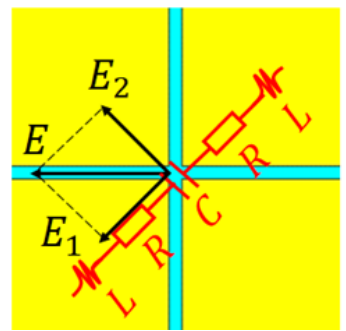

(c)

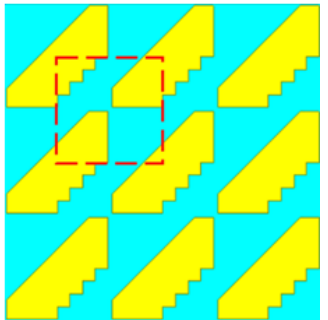

(b)

(d)

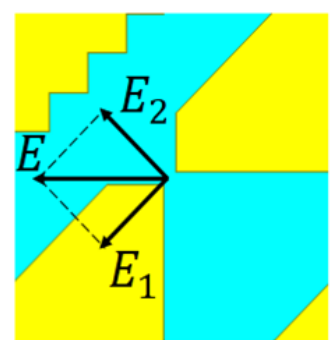

Fig. 2. The structure of the dashed box unit.

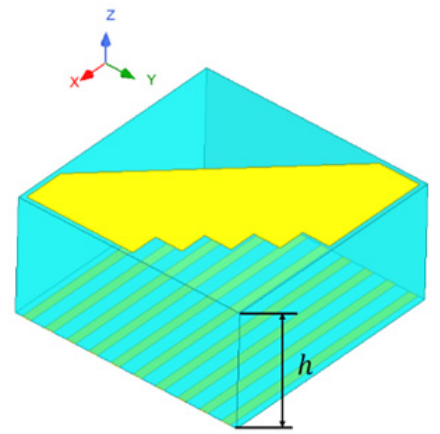

(a)

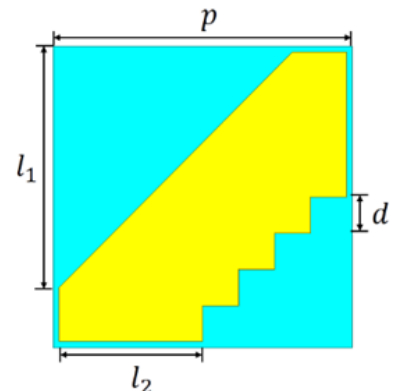

(b)

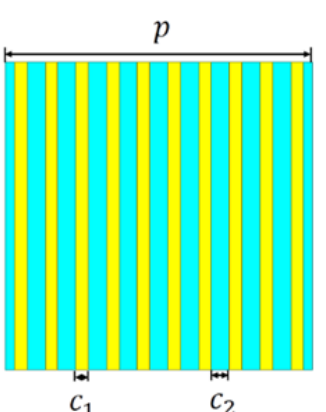

(c)
Fig. 3. The structure diagrams of unit.

When this unit is placed above the linear polarized antenna, if the upper and lower surfaces have the same metal structure, a part of the incident wave radiated by the linear polarized antenna will be converted into a cross-polarized reflected wave and be reflected back. The reflected crosspolarized wave will be reflected with the same polarization by the bottom metal plate of the antenna, and it will be incident on the PRS again. In the forward radiation space of the unit, there are both left-handed circularly polarized waves and right-handed circularly polarized waves, it will seriously affect the circular polarization performance of the metasurface. Therefore, when the unit is placed above a microstrip antenna to form an F-P resonant cavity antenna, special attention should be paid to the cross-polar-

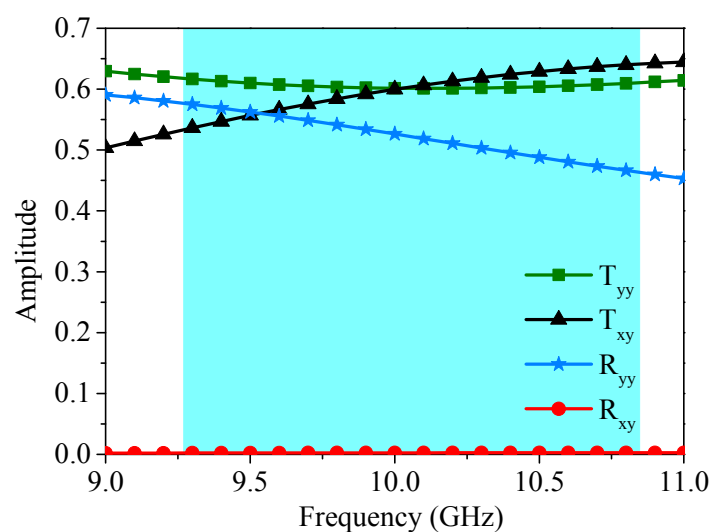

(a)

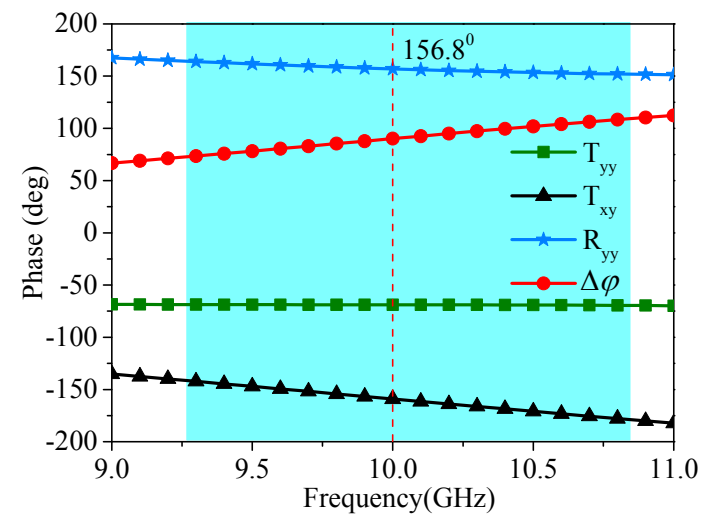

(b)

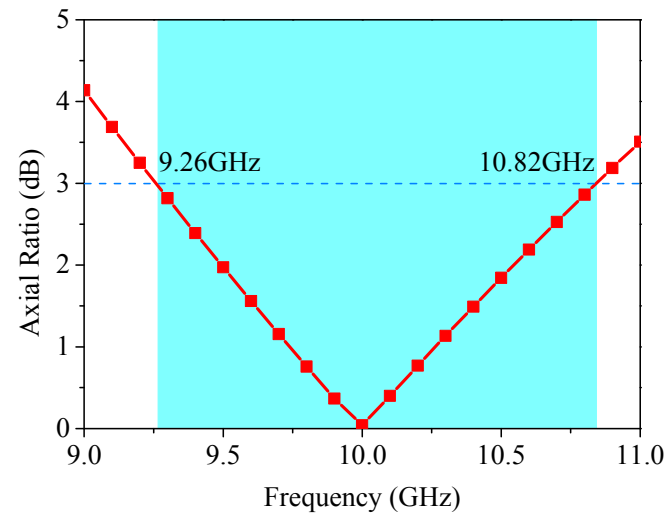

(c)

Fig. 4. Simulation results of the unit: (a) Reflection and transmission amplitude; (b) Reflection and transmission phase; (c) AR.

ized reflection coefficient of the PRS, and the cross-polarized wave in the resonant cavity should be minimized.

In order to reduce the cross-polarized wave in the resonant cavity, a metasurface which is composed of a zigzag patch and a polarization grid is proposed, as shown in Fig. 3. The upper layer is a zigzag metal structure, the middle is a dielectric layer, and the bottom layer is a metal grid. The dielectric layer uses F4B with a dielectric constant of 2.65 and a loss tangent of 0.001 . The parameters of the unit are as follows: $p=5 \mathrm{~mm}, d=0.6 \mathrm{~mm}, h=2.5 \mathrm{~mm}$, $l_{1}=3.9 \mathrm{~mm}, l_{2}=2.4 \mathrm{~mm}, c_{1}=0.2 \mathrm{~mm}, c_{2}=0.3 \mathrm{~mm}$.

ANSYS 2019 is used to simulate the designed unit. When the $y$-polarized wave is incident along the $+z$-axis, 
the simulation results are shown in Fig. 4. $T_{\mathrm{yy}}$ represents the co-polarized transmission component, $T_{\mathrm{xy}}$ represents the cross-polarized transmission component, $R_{\mathrm{yy}}$ represents the co-polarized reflection component, $R_{\mathrm{xy}}$ represents the crosspolarized reflection component, and $\Delta \varphi$ represents the phase difference of the $T_{\mathrm{yy}}$ and $T_{\mathrm{xy}}$. It can be seen from the results that the design of the polarization grid makes the reflected wave almost no cross-polarization component. Comparing the $T_{\mathrm{yy}}$ and $T_{\mathrm{xy}}$, the amplitude is basically equal, the value of phase difference is $90^{\circ}$ and the phase of the $T_{\mathrm{yy}}$ is ahead of the phase of the $T_{\mathrm{xy}}$. It can be concluded that when the $y$-polarized incident wave is incident alongthe direction of $+z$-axis, the transmitted wave is a left-handed circularly polarized wave. The Axial Ratio (AR) of the circularly polarized transmitted wave can be calculated by (14) [20]. As shown in Fig. 4, the 3-dB bandwidth of AR is 9.26 10.84 GHz.

$$
\mathrm{AR}=\left|20 \lg \left\{\tan \left[0.5 \arcsin \left(\frac{2 T_{\mathrm{yy}} T_{\mathrm{xy}}}{T_{\mathrm{yy}}^{2}+T_{\mathrm{xy}}^{2}} \sin \Delta \varphi\right)\right]\right\}\right|
$$

Rotate the zigzag metal patch $90^{\circ}$ clockwise around $\boldsymbol{z}$-axis and other parameters are unchanged to form a mirror image unit, as shown in Fig. 5(a). When the $y$-polarized incident wave is incident along the $+z$-axis, the simulation results are shown in Fig. 5. $T_{\text {yy }}^{\prime}$ represents the co-polarized transmission component, $T_{\mathrm{xy}}^{\prime}$ represents the cross-polarized transmission component, $R_{\text {yy }}^{\prime}$ represents the co-polarized reflection component, and $\Delta \varphi^{\prime}$ represents the phase difference of $T_{\mathrm{yy}}^{\prime}$ and $T_{\mathrm{xy}}^{\prime}$. It can be seen from the result that

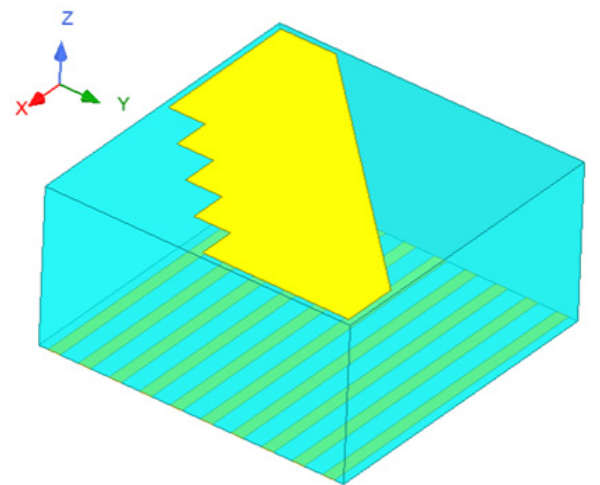

(a)

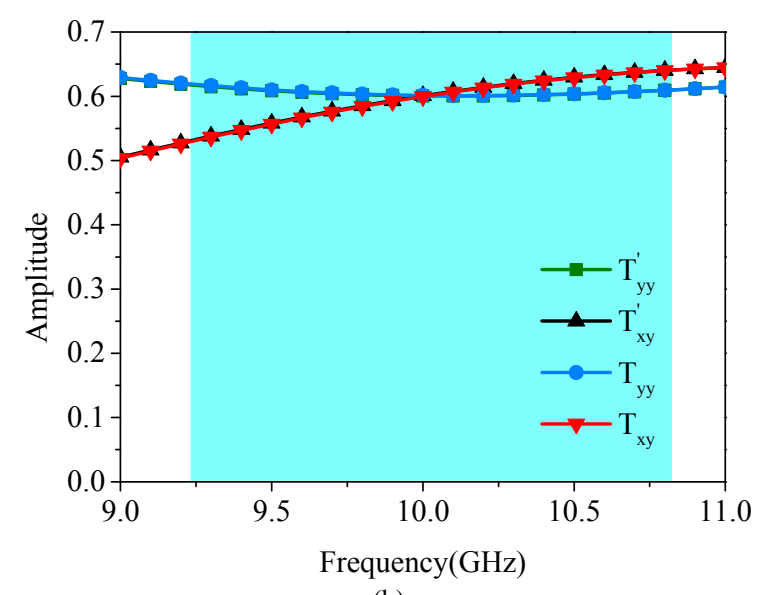

(b)

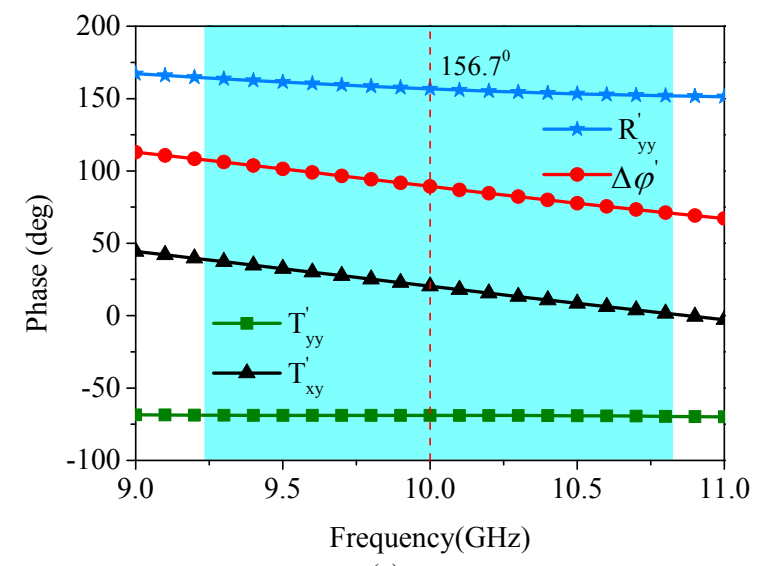

(c)

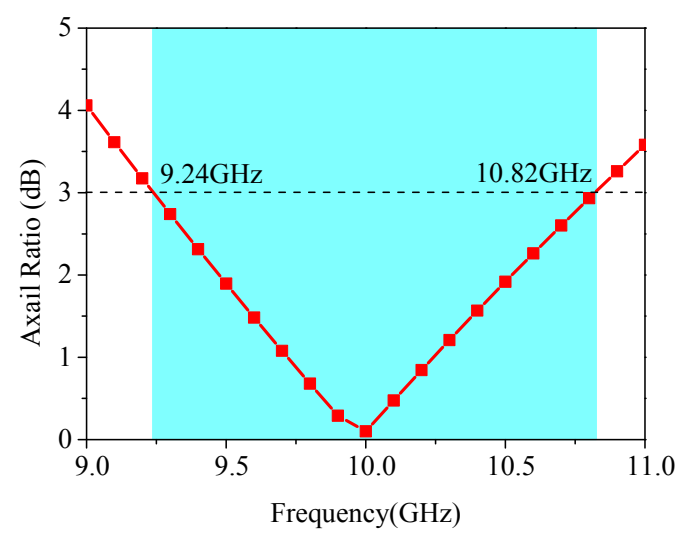

(d)

Fig. 5. Simulation results of mirror image unit: (a) Structure diagram of mirror image unit; (b) reflection amplitude and transmission amplitude; (c) reflection phase and transmission phase; (d) AR.

3-dB bandwidth of AR is 9.24 10.82 GHz. The amplitude of $T_{\text {xy }}^{\prime}$ and $T_{\text {yy }}^{\prime}$ is equal to the original unit. The value of phase difference between $T_{\text {xy }}^{\prime}$ and $T_{\text {yy }}^{\prime}$ is $90^{\circ}$. The phase of $T_{\mathrm{xy}}^{\prime}$ is ahead of $T_{\mathrm{yy}}^{\prime}$. When the $y$-polarized incident wave is incident on the mirror image unit along the direction of $+z$-axis, the transmitted wave is a right-handed circularly polarized wave.

\section{Low RCS and High Gain Linear Polarization Antenna Based on Metasurface}

Assuming $\mathbf{E}_{1}$ and $\mathbf{E}_{2}$ are circularly polarized waves that propagating along the direction of $+z$-axis with equal amplitude and opposite rotation directions:

$$
\begin{aligned}
& \mathbf{E}_{\mathbf{1}}=\frac{E_{0}}{2}\left(\mathbf{e}_{\mathrm{x}}-\mathrm{j} \mathbf{e}_{\mathrm{y}}\right) \mathrm{e}^{-\mathrm{j} k z}, \\
& \mathbf{E}_{\mathbf{2}}=\frac{E_{0}}{2}\left(\mathbf{e}_{\mathrm{x}}+\mathrm{j} \mathbf{e}_{\mathrm{y}}\right) \mathrm{e}^{-\mathrm{j} k z}, \\
& \mathbf{E}_{\mathbf{1}}+\mathbf{E}_{\mathbf{2}}=\mathbf{e}_{\mathrm{x}} E_{0} \mathrm{e}^{-\mathrm{j} k z} .
\end{aligned}
$$




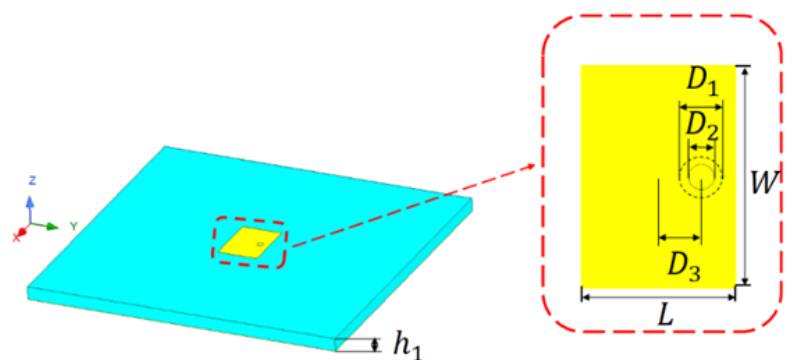

(a)

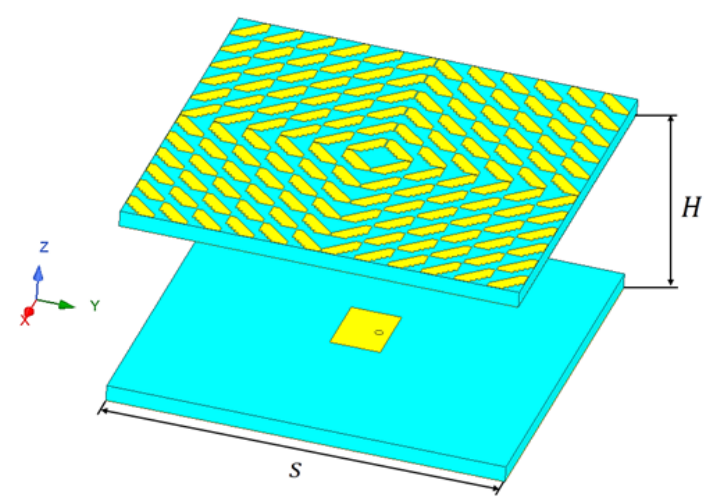

(b)

Fig. 6. The structure diagram of the antenna: (a) Structure diagram of microstrip antenna; (b) structure diagram of F-P resonant cavity antenna.

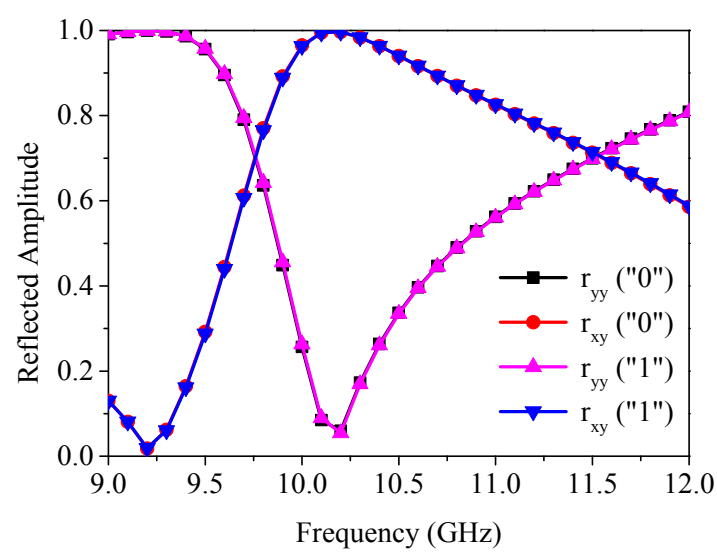

(a)

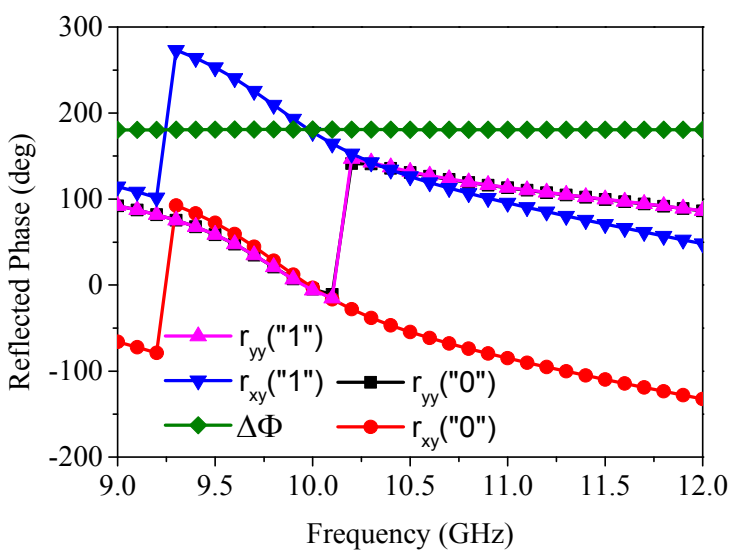

(b)

Fig.7. Reflection characteristics of metasurface unit: (a) Reflection amplitude; (b) Reflection phase.
It can be seen from the above equations that two circularly polarized waves with the same amplitude and opposite rotation directions can be combined into a linearly polarized wave.

Based on the theory, the original unit and mirror image unit were arranged in a checkerboard to form a $12 \times 12$ PRS and placed above the microstrip antenna which working at $10 \mathrm{GHz}$ to form a high-gain linear polarization F-P resonator cavity antenna, as shown in Fig. 6.

The reflection characteristics of the two types of units when the $y$-polarized wave is incident along the direction of $+z$-axis are shown in Fig. 7 . " 0 " represents the original unit, " 1 " represents the mirror image unit. $r_{\mathrm{xy}}$ represents the cross-polarized reflected wave, $r_{\mathrm{yy}}$ represents the co-polarized reflected wave, $\Delta \Phi$ represents the phase difference of the $r_{\mathrm{xy}}(" 0 ")$ and $r_{\mathrm{xy}}(" 1 ")$. It can be seen from Fig. 7 that the cross-polarized reflected wave of the two units satisfies the condition of phase cancellation. That is to say, the designed antenna has the RCS reduction effect, and the amplitude of $r_{\mathrm{xy}}$ is largest at $10.3 \mathrm{GHz}$, so the RCS reduction value would be the largest. The height of the air layer can be calculated by (10). After optimization, the height is $H=29.5 \mathrm{~mm}$. The other parameters of the antenna are as follows: $h_{1}=2.5 \mathrm{~mm}, L=7.6 \mathrm{~mm}, W=10.9 \mathrm{~mm}, D_{1}=3 \mathrm{~mm}$, $D_{2}=1.2 \mathrm{~mm}, D_{3}=2 \mathrm{~mm}, s=60 \mathrm{~mm}$.

\subsection{Radiation Performance Analysis}

The results of simulation are shown in Fig. 8(a). The $-10-\mathrm{dB}$ bandwidth of the microstrip antenna is between 9.61 and $10.40 \mathrm{GHz}$. After loading the metasurface, the bandwidth of the antenna is reduced, the $-10-\mathrm{dB}$ bandwidth is $9.50 \sim 10.04 \mathrm{GHz}$, and the resonance point of the antenna is shifted to low frequency.

It can be seen from Fig. 8(b) that the AR of the F-P resonator cavity antenna is more than $40 \mathrm{~dB}$ in the range of $9.50 \sim 10.04 \mathrm{GHz}$ and larger than that of the microstrip antenna in the range of the $9.55 \sim 9.85 \mathrm{GHz}$. This indicates that the linear polarization purity of the antenna has been improved after adding the checkerboard metasurface.

Compare the gain pattern of two antennas at the $10 \mathrm{GHz}$. As is shown in Fig. 8(c) and Fig. 8(d), it can be seen that the maximum gain of the F-P resonator cavity antenna is improved, the directivity of antenna is enhanced, and the metasurface has the effect of beam focus. It can be seen from Fig. 8(e) that the gain of the F-P resonator cavity antenna is improved in the range of $9.50 \sim 10.04 \mathrm{GHz}$, and the maximum gain increases $5.55 \mathrm{~dB}$, but the backward gain also increases. This is due to the partial reflection characteristics of the metasurface. Some of the EM waves radiated by the microstrip antenna are reflected by the PRS, and the reflected EM waves will be diffracted through the antenna bottom plate. Therefore, the backward gain is improved.

Based on the above analysis, the designed F-P resonant cavity antenna can improve the polarization purity and enhance forward gain in the range of 9.50 10.04 GHz. 


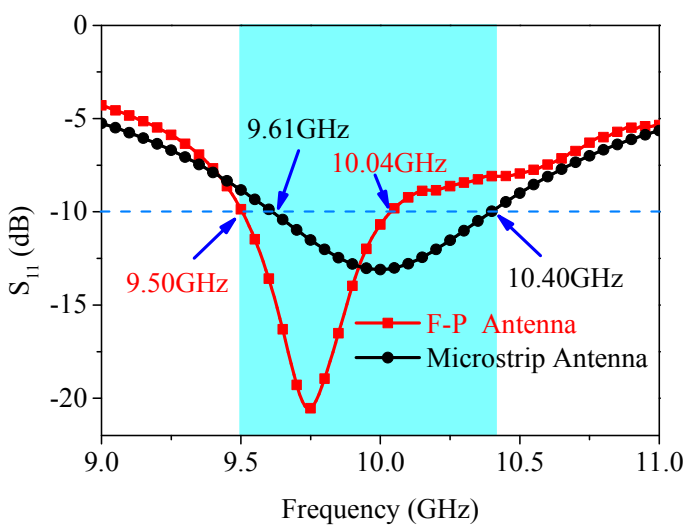

(a)

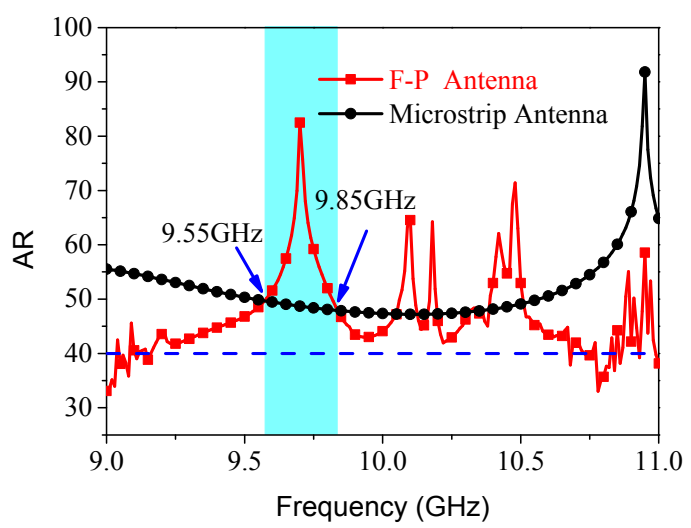

(b)

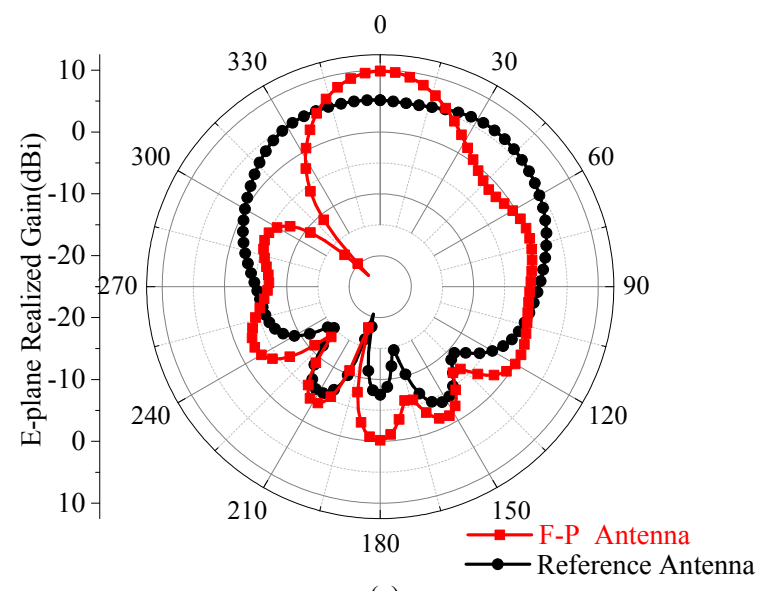

(c)

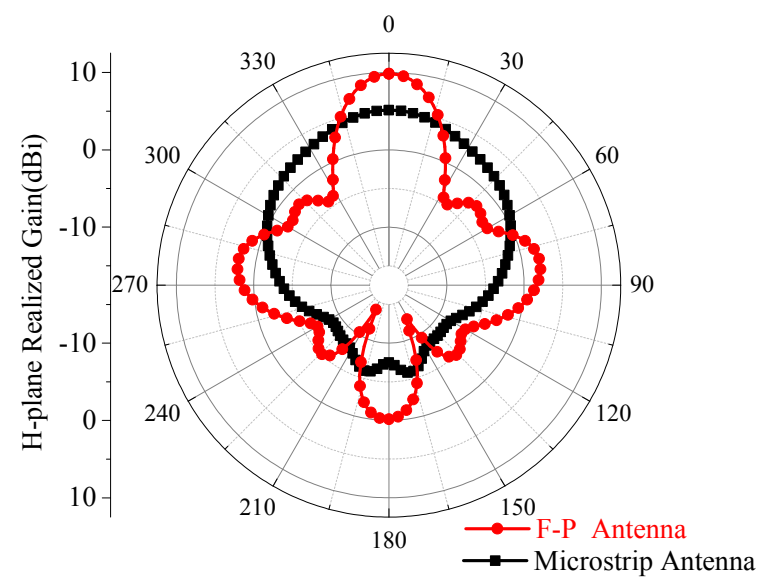

(d)

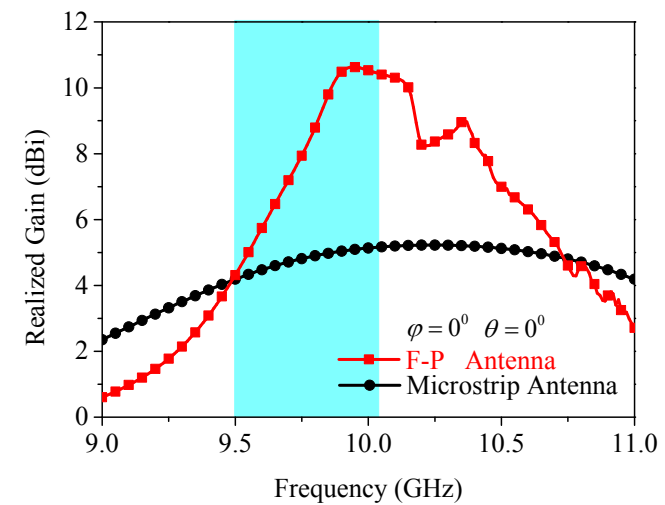

(e)

Fig. 8. Comparison of radiation performance: (a) Working bandwidth; (b) AR; (c) gain pattern of $E$ plane at $10 \mathrm{GHz}$; (d) gain pattern of $\mathrm{H}$ plane at $10 \mathrm{GHz}$; (e) gain of maximum radiation direction.

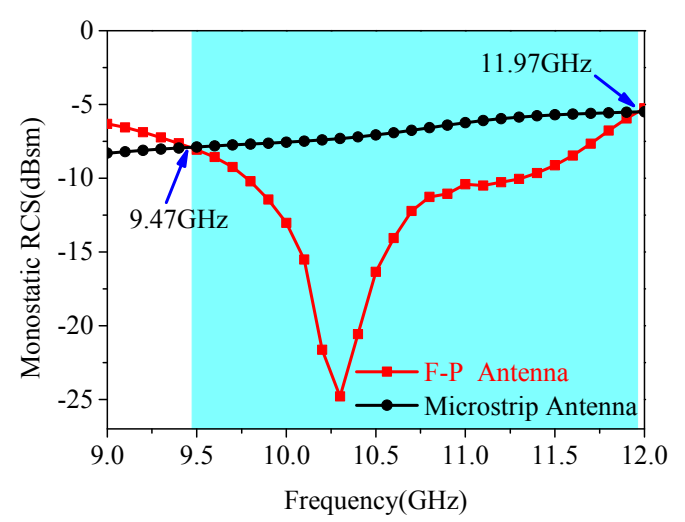

(a)

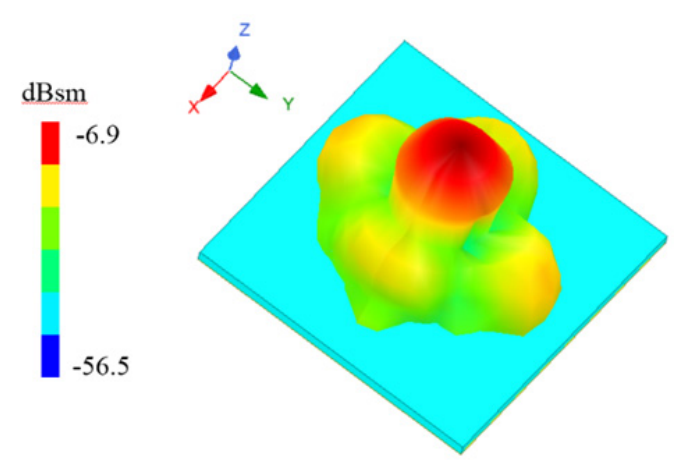

(b)

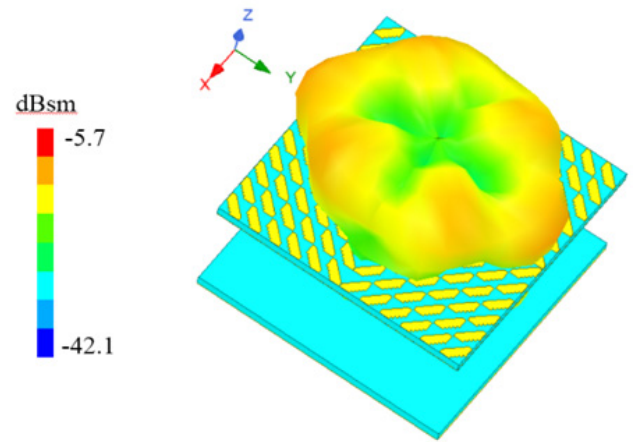

(c)

Fig. 9. Scattering performance of the antenna: (a) Monostatic RCS; (b) three-dimensional RCS diagram of microstrip antenna at $10.3 \mathrm{GHz}$; (c) three-dimensional RCS diagram of F-P resonator antenna at $10.3 \mathrm{GHz}$. 


\subsection{Scattering Performance Analysis}

Employ the $y$-polarized incident wave as the radar detection wave, and analyze the scattering performance of the F-P resonator cavity antenna. The simulation results are shown in Fig. 9. It can be seen from Fig. 9(a) that the F-P resonant cavity antenna has achieved monostatic RCS reduction in the range of $9.47 \sim 11.97 \mathrm{GHz}$, and the frequency point of the largest reduction value is $10.3 \mathrm{GHz}$. The largest reduction value is $17.47 \mathrm{~dB}$. It also verifies the previous presumption about the low scattering performance of the antenna. The three-dimensional RCS diagrams of the antennas at $10.3 \mathrm{GHz}$ are shown in Fig. 9(b) and Fig. 9(c). It can be seen that the F-P resonant cavity antenna has an obvious RCS reduction effect compared to the microstrip antenna.

\section{The Result Analysis of Measurement}

As shown in Fig. 10, in order to verify the simulation results, the F-P resonator cavity antenna was processed. During processing, the intermediate dielectric layer of the microstrip antenna and the metasurface were widened, and four via holes with a diameter of $3 \mathrm{~mm}$ were punched to facilitate the combination of the microstrip antenna and the metasurface. Nylon screw was used to fix the antenna and strictly control the height of the air cavity to form an F-P resonant cavity antenna.

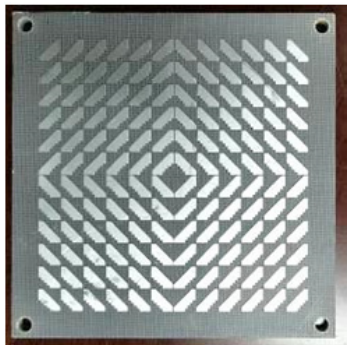

(a)

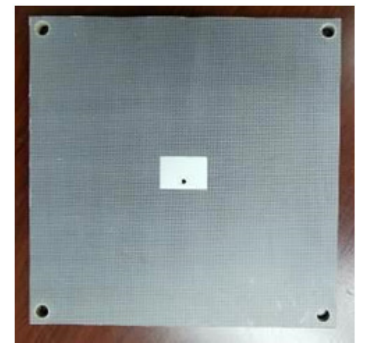

(b)

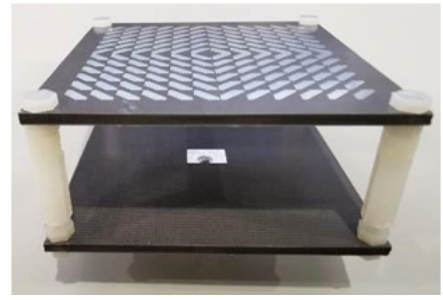

(c)

Fig. 10. Finished product :(a) Metasurface; (b) microstrip antenna; (c) F-P resonant cavity antenna.

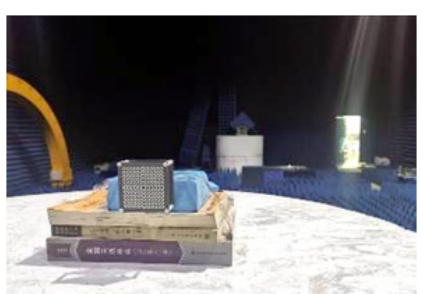

(a)

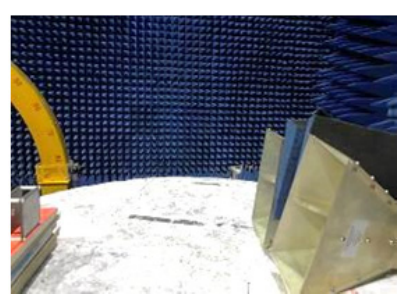

(b)
Fig. 11. Measured environment: (a) Radiation; (b) scattering.
The environment of microwave anechoic chamber is shown in Fig. 11, and this experiment uses the vector network analyzer of Agilent 5230C. The standard horn antenna is used as the transmitting antenna, and the test antenna is placed on the turntable. The test antenna was aligned with the center of the standard horn antenna by a level calibrator. The distance between the test antenna and the standard horn antenna satisfies the far-field conditions. The reflection coefficient and direction pattern were tested respectively, and the scattering characteristic of the F-P resonator cavity antenna was tested. The comparison between the simulation results and the measured results is shown in Fig. 12. The difference between the simulation results and measured results is mainly due to the environment error and the machining error, and the difference is within the allowable range of error.

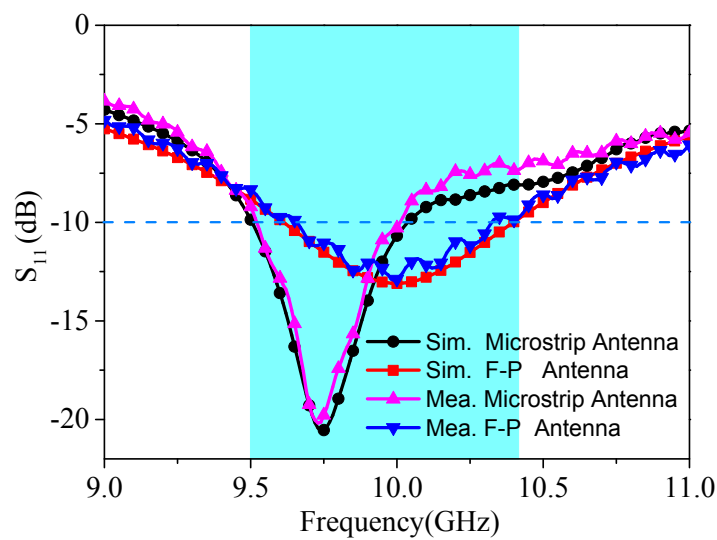

(a)

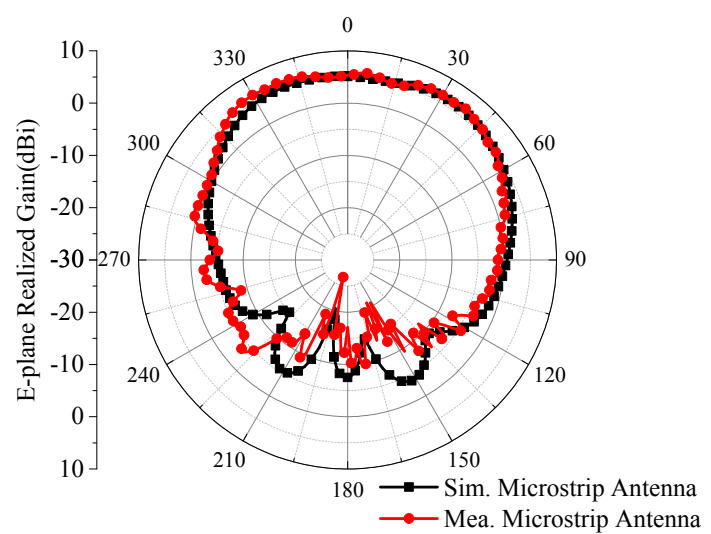

(b)

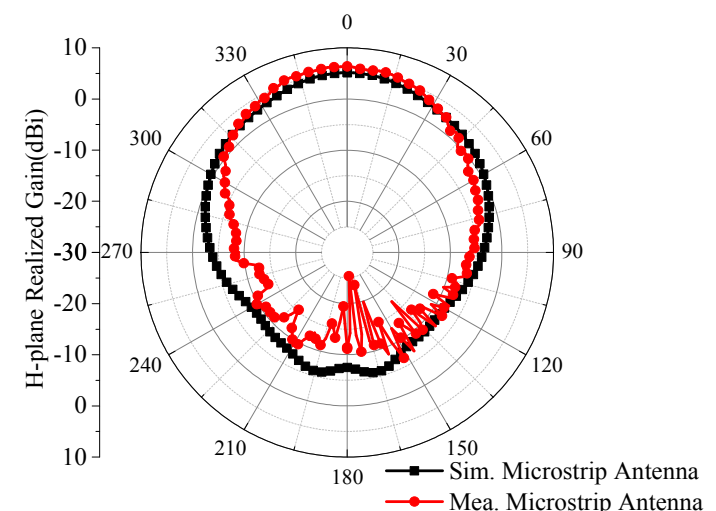

(c) 


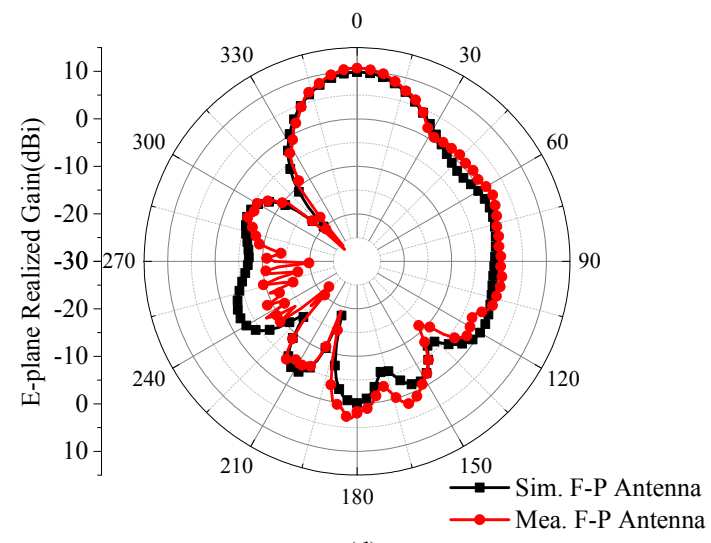

(d)

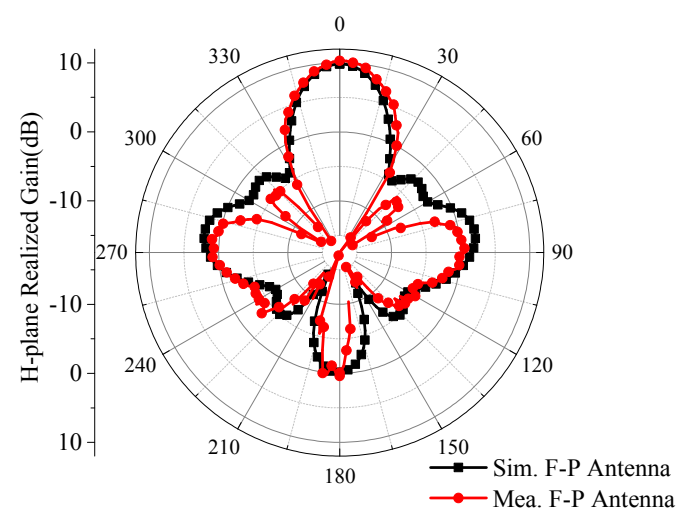

(e)

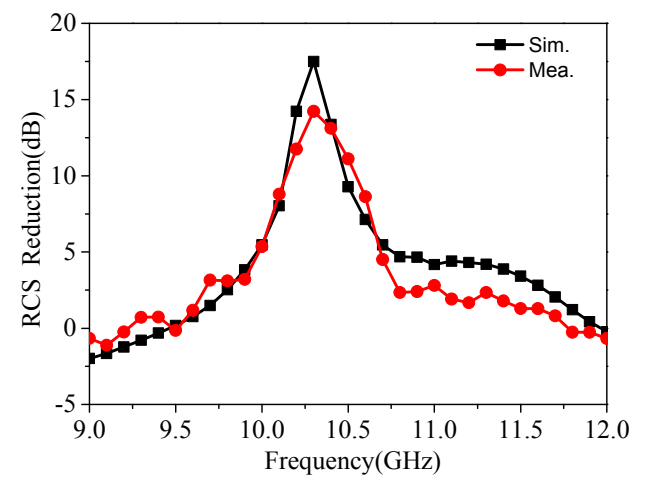

(f)

Fig. 12. Comparison between simulation results and measurement results: (a) Working bandwidth; (b) E-plane gain pattern of microstrip antenna at $10 \mathrm{GHz}$; (c) H-plane gain pattern of microstrip antenna at $10 \mathrm{GHz}$; (d) E-plane gain pattern of F-P resonant cavity antenna at $10 \mathrm{GHz}$; (e) H-plane gain pattern of F-P resonant cavity antenna at $10 \mathrm{GHz}$; (f) $\mathrm{RCS}$ reduction.

\section{Conclusion}

In this paper, a transmissive linear-circular polarization conversion metasurface is designed. The polarization conversion metasurface unit and its mirror image unit were arranged in a checkerboard to form a $12 \times 12$ polarization conversion metasurface, which are placed on the top of the microstrip antenna as a cladding layer. Because two circular polarization waves with the same amplitude and opposite rotation can be used to synthesize a linear polarization wave, then a linear polarization F-P resonant cavity antenna is formed. The F-P resonant cavity antenna also has high gain and low RCS. This design can realize the comprehensive control of radiation and low scattering. In order to verify the feasibility of the design, the F-P resonant cavity antenna was processed, and measured in a microwave anechoic chamber. The measured results and simulated results have shown good consistency. This design has important reference value in the comprehensive control of antenna's radiation performance and scattering performance.

\section{Acknowledgements}

This work was supported by the National Natural Science Foundation of China (No. 6217012409, 61801508), the Natural Science Basic Research Program of Shaanxi Province, China (No. 2020JM-350, 20200108, 20210110 and 2020022) and the Postdoctoral Innovative Talents Support Program of China (No. BX20180375, 2019M653960).

\section{References}

[1] CUI, T. J. Electromagnetic metamaterials-from effective media to field programmable systems. Scientia Sinica Informationis, 2020, vol. 50, no. 10 , p. 1427-1461. (In Chinese) DOI: 10.1360/SSI2020-0123

[2] LI, S. J., LI, Y. B., ZHANG, L., et al. Programmable controls to scattering properties of a radiation array. Laser \& Photonics Reviews, 2021, vol. 15, no. 2. DOI: 10.1002/lpor.202000449

[3] LI, S. J., LI, Y. B., LI, H., et al. A thin self-feeding Janus metasurface for manipulating incident waves and emitting radiation waves simultaneously. Annalen der Physik, 2020, vol. 532, no. 5. DOI: $10.1002 /$ andp. 202000020

[4] HAN, B. W., LI, S. J., LI, Z. Y., et al. Asymmetric transmission for dual-circularly and linearly polarized waves based on a chiral metasurface. Optics Express, 2021, vol. 29, no. 13, p. 19643 to 19654. DOI: 10.1364/OE.425787

[5] HAN, B. W., LI, S. J., CAO, X. Y., et al. Dual-band transmissive metasurface with liner to dual-circular polarization conversion simultaneously. AIP Advances, 2020, vol. 10, p. 1-9. DOI: $10.1063 / 5.0034762$

[6] GAO, K., CAO, X. Y., GAO, J., et al. Characteristic mode analysis of wideband high-gain and low-profile metasurface antenna. Chinese Physics B, 2021, vol. 30, no. 6, p. 1-7. DOI: 10.1088/1674-1056/abdb23

[7] LI, T., YANG, H. H., LI, Q., et al. Broadband low RCS and high gain microstrip antenna based on concentric ring-type metasurface. IEEE Transactions on Antennas and Propagation, 2021, vol. 69, no. 9, p. 5325-5334. DOI: 10.1109/TAP.2021.3061095

[8] YANG, H. H., LI, T., XU, L., et al. Low in-band-RCS antennas based on anisotropic metasurface using a novel integration method. IEEE Transactions on Antennas and Propagation, 2021 vol. 69, no. 3, p. 1239-1248. DOI: 10.1109/TAP.2020.3016161

[9] YANG, H. H., CAO, X. Y., YANG, F., et al. A programmable metasurface with dynamic polarization, scattering and focusing control. Scientific Reports, 2016, vol. 6, p. 1-11. DOI: $10.1038 /$ srep35692 
[10] ZHENG, Q., GUO, C., DING, J., et al. A broadband low-RCS metasurface for CP patch antennas. IEEE Transactions on Antennas and Propagation, 2021, vol. 69, no. 6, p. 3529-3534. DOI: 10.1109/TAP.2020.3030547

[11] HAO, B., YANG, B. F., GAO, J., et al. A coding metasurface antenna array with low radar cross section. Acta Physica Sinica, 2020, vol. 69, no. 24, p. 1-11. (In Chinese) DOI: 10.7498/aps.69.20200978

[12] LIU, Y., JIA, Y., ZHANG, W., et al. An integrated radiation and scattering performance design method of low-RCS patch antenna array with different antenna elements. IEEE Transactions on Antennas and Propagation, 2019, vol. 67, no. 9, p. 6199-6204. DOI: 10.1109/TAP.2019.2925194

[13] PAZOKIAN, M., KOMJANI, N., KARIMIPOUR, M. Broadband RCS reduction of microstrip antenna using coding frequency selective surface. IEEE Antennas and Wireless Propagation Letters, 2018, vol. 17, no. 8, p. 1382-1385. DOI: 10.1109/LAWP.2018.2846613

[14] LIU, Y., LI, N., JIA, Y., et al. Low RCS and high-gain patch antenna based on a holographic metasurface. IEEE Antennas and Wireless Propagation Letters, 2019, vol. 18, no. 3, p. 492-496. DOI: 10.1109/LAWP.2019.2895117

[15] ZHENG, Y., GAO, J., ZHOU, Y., et al. Wideband gain enhancement and RCS reduction of Fabry-Perot resonator antenna with chessboard arranged metamaterial superstrate. IEEE Transactions on Antennas and Propagation, 2017, vol. 66, no. 2, p. 590-598. DOI: 10.1109/TAP.2017.2780896

[16] ZHANG, L., WAN, X., LIU, S., et al. Realization of low scattering for a high-gain Fabry-Perot antenna using coding metasurface. IEEE Transactions on Antennas and Propagation, 2017, vol. 65, no. 7, p. 3374-3383. DOI: 10.1109/TAP.2017.2700874

[17] XIE, P., WANG, G. M., LI, H. P., et al. Circularly polarized Fabry-Perot antenna employing a receiver-transmitter polarization conversion metasurface. IEEE Transactions on Antennas and Propagation, 2020, vol. 68, no. 4, p. 3213-3218. DOI: 10.1109/TAP.2019.2950811

[18] DONG, J., DING, C., MO, J. J., et al. A low-profile wideband linear-to-circular polarization conversion slot antenna using metasurface. Materials, 2020, vol. 13, p. 1-12. DOI: $10.3390 / \mathrm{ma1} 3051164$

[19] LIU, Y., HUANG, Y. X., LIU, Z. W., et al. Design of a compact wideband $\mathrm{CP}$ metasurface antenna. International Journal of $R F$ and Microwave Computer-Aided Engineering, 2020, vol. 30, no. 10. DOI: $10.1002 /$ mmce. 22332

[20] GUO, Z. X., CAO, X. Y., GAO, J., et al. A novel composite transmission metasurface with dual functions and its application in microstrip antenna. Journal of Applied Physics, 2020, vol. 127, p. 1-9. DOI: $10.1063 / 1.5143147$

\section{About the Authors...}

Jia LU was born in Shaanxi province, China. He received his B.S. degree from the Air Force Engineering University, Xi'an, China, in 2015. He currently works towards his M.S. degree. In his research, he specializes in metamaterial, antenna design and RCS reduction techniques.

Xiangyu CAO received her M.S. degree from the Air Force Missile Institute in 1989. In the same year, she joined the Air Force Missile Institute. She received her Ph.D. degree in the Missile Institute of AFEU in 1999. From 1999 to 2002, she was engaged in postdoctoral research in Xidian University, China. She was a Senior Research Associate in the Department of Electronic Engineering, City University of Hong Kong from June 2002 to Dec. 2003. She is currently a Professor and a senior member of IEEE. Her research interests include computational electromagnetic, electromagnetic metamaterials and their antenna applications.

Jun GAO received the M.S. and Ph.D. degrees from the Air Force Missile Institute in 1984 and 1987, respectively. He joined the Air Force Missile Institute in 1987 as an assistant-teacher. He became an Associate Professor in 2000. He is currently a Professor of the Information and Navigation College, Air Force Engineering University of CPLA. He has authored and coauthored more than 100 technical journal articles and conference papers, and holds one China soft patent. His research interests include smart antennas, electromagnetic metamaterial and their antenna applications.

Huanhuan YANG (S'15-M'19) received the M.S. and Ph.D. degrees from the Air Force Engineering University (AFEU), Xi'an, China, in 2012 and 2016, respectively. He was a joint-supervision Ph.D. student of AFEU and Tsinghua University. He is currently a Lecturer and also a postdoctoral researcher in AFEU. His research interests include advanced antenna array, reconfigurable antenna, reflectarray, metasurface and RCS reduction technique.

Liaori JIDI was born in Sichuan province, China. $\mathrm{He}$ received his B.S. degree from the Electronic and Information College, Tongji University in 2016, and he was the commencement student speaker of Tongji University in 2016. In 2018, he received his M.S. degree from the Air Force Engineering University (AFEU). He currently works towards his Ph.D. degree. In his research, he specializes in metamaterial, antenna array design, RCS reduction techniques and SSPPs.

Kun GAO was born in Henan province, China. He received his B.S. degree from the Air Force Engineering University, Xi'an, China, in 2019. He currently works towards his M.S. degree. In his research, he specializes in characteristic mode analysis, metamaterial, and RCS reduction techniques. 